\section{Bleach Usage for CJD Disinfection}

\section{To the Editor:}

The recent report by Bastian and Jennings ${ }^{1}$ provided a timely and useful procedure for disinfecting surfaces and equipment contaminated by Creutzfeldt-Jakob disease (CJD) materials. However, several questions regarding bleach usage are unanswered in the protocol.

Since no specific strength of bleach was indicated, was full-strength household bleach $(5.25 \%$ sodium hypochlorite) used? If so, given the corrosiveness of bleach, I am surprised that the instruments could be soaked for one hour without showing any damage. In a series of tests to determine a safe concentration and exposure time for surgical instruments, we found that corrosion of some instruments began after ten minutes in a 1:10 dilution of bleach $(0.5 \%$ sodium hypochlorite). This is the concentration recommended for a one-hour submersion of materials by Brown et al, ${ }^{2}$ who determined that $0.5 \%$ and $1.3 \%$ sodium hypochlorite produced the inactivation of CJD isolates in 15 minutes.

It was also unclear from the report whether or not the equipment and surfaces swabbed with full-strength bleach subsequently were rinsed with water or simply were allowed to air dry.

Some clarification of these points is necessary to make this protocol more practical.

\section{REFERENCES}

1. Bastian FO, Jennings RA: Creutzfeldt-Jakob disease: Procedures for handling diagnostic and research materials. Infect Control 1984; 5:48-50.

2. Brown P, Gibbs CH Jr, Amyx DUM, et al: Chemical disinfection of Creutzfeldt-Jakob disease virus. $N$ Fingl / Mud 1982; 306: $1299-1312$.

\section{Andrea Scheidt, MPH}

Hospital Sanitarian

The New York Hospital

New York, New York

Frank O. Bastian, MD, was given the opportunity to respond to Ms. Scheidt's comments.

Appropriate decontamination can be achieved by soaking stainless steel surgical instruments for one hour in a $0.5 \%$ solution of sodium hypochlorite. This, however, will remove the highly polished surface of the instruments with subsequent corrosion and rust formation. We recommend the collection of instruments in a stainless steel pan with a lid which is sealed in a bioclavable bag and transported to an autoclave for sterilization.

Equipment and surfaces exposed to CJD biological materials are swabbed with full-strength bleach, allowed to air dry, and then rinsed with water.

Frank O. Bastian, MD Associate Professor Department of Pathology University of South Alabama Mobile, Alabama

\section{Homestudy Course: "Principles of Epidemiology"}

\section{To the Editor:}

Infection Control Vol. 5, No. 1, January 1984 contains an article by Sue Crow entitled "Training of Personnel for Infection Control," which states that the CDC has developed a homestudy course in hospital epidemiology.

This statement is incorrect and we would appreciate the correct information being printed in the next issue of

\section{Infection Control.}

The CDC has a homestudy course called "Principles of Epidemiology," course 3030-G, designed to teach principles and skills in the surveillance and investigation of acute, communicable diseases. The intended audience is persons performing epidemiologic functions in State and local health agencies.

The principles and skills may be applied to the practice of epidemiology in hospital environments. However, no special emphasis is given to hospital settings in the teaching exercises.

Many nurses enroll in this homestudy course and we encourage their participation. However, it should be understood that this is not a course in hospital epidemiology.

Information regarding enrollment may be obtained by writing Mr. Jack Stanley, Chief, Homestudy Services Branch, Division of Instructional Services, Center for Professional Development and Training, Centers for Disease Control, Atlanta, GA 30333.

The CDC does offer two classroom courses in hospital infections; they are "Surveillance, Prevention and Control of Nosocomial Infections" and "Management Skills for Infection Control Nurses." Information regarding these courses may be obtained from Ms. Nancy Adams, Registrar, Support Services Branch, Division of Instructional Services, Center for Professional Development and Training, Centers for Disease Control, Atlanta, GA 30333 .

Conrad P. Ferrara

Chief, Support Services Branch Division of Instructional Services Center for Professional Development and Training Centers for Disease Control Atlanta, Georgia 